der Untersuchungen noch nicht auf Einzelheiten eingegangen werden. Lediglich im Zusammenhang mit dem oben angegebenen Diffusionskoeffizienten von ${ }^{210} \mathrm{Bi}$ in den beiden Blei-Zinn-Legierungen wäre zu bemerken, daß das Diffusionsverhalten solcher in Spuren vorhandener Beimengungen in E-System-Legierungsschmelzen von dem Verteilungsverhalten auf die verschiedenen Nahordnungsbereiche abhängen wird, so daß kein einheitliches Verhalten erwartet werden kann.

Herrn Professor Dr. H. Condes danken wir für sein förderndes Interesse. Die Untersuchungen wurden ermöglicht durch Unterstützung seitens der Deutschen Forschungsgemeinschaft sowie des Bundesministeriums für wissenschaftliche Forschung.

\title{
Zum Mechanismus der Dotierung von Silicium mit Phosphor bei Anwesenheit einer Oberflächenschicht aus $\left(\mathrm{SiO}_{2}\right)_{n} \cdot \mathrm{P}_{2} \mathrm{O}_{5}$
}

\author{
Helga Garski * \\ Siemens \& Halske AG, Wernerwerk für Halbleiter, München \\ (Z. Naturforschg. 22 a, 66-75 [1967] ; eingegangen am 8. September 1966)
}

\begin{abstract}
The diffusion of phosphorus into silicon out of gaseous $\mathrm{P}_{2} \mathrm{O}_{5}$ is rated by a glassy surface layer of $\left(\mathrm{SiO}_{2}\right)_{n} \cdot \mathrm{P}_{2} \mathrm{O}_{5}$. The dependence of glass composition and sheet resistance of the diffused layer on $\mathrm{P}_{2} \mathrm{O}_{5}$-concentration in the reaction zone is examined at constant diffusion time and temperature. There is no simple linear relation. The results can be explained by a discussion of the phase diagram of $\mathrm{SiO}_{2}-\mathrm{P}_{2} \mathrm{O}_{5}$.

Even at low doping levels far below the solubility limit only part of all phosphorus in the silicon contributes to electrical conduction. The relative part of inactive phosphorus increases with decreasing resistivity.
\end{abstract}

Zur Diffusion von Phosphor in Silicium sind eine Reihe verschiedener Verfahren üblich, bei denen ein Trägergas mit dem Dampf einer geeigneten Phosphorverbindung beladen wird und über die auf hoher Temperatur befindlichen Siliciumproben strömt. Der Mechanismus des Einbaues von Phosphor ins Silicium im Verlauf der Behandlung ist ein anderer, je nachdem, ob die Diffusion in Gegenwart von Sauerstoff erfolgt - sei es als Trägergas und/oder chemisch gebunden in der Quellensubstanz - oder nicht.

Eine sauerstoff-freie Diffusion ist in inertem Trägergas realisierbar durch Verdampfen von elementarem Phosphor oder von geeigneten, bei der Diffusionstemperatur leicht zersetzlichen Phosphorverbindungen. In diesem Fall wird der Einbau ins Silicium beherrscht durch ein dynamisches Gleichgewicht zwischen in der Festkörper-Oberfläche gelösten und im Gasraum befindlichen P-Atomen (bzw. Molekülen). Der Mechanismus der Diffusion ohne Anwesenheit

* Jetzt: Institut für Gesteinshüttenkunde, Rhein.-Westf. Techn. Hochschule Aachen.

1 F. M. Smits u. R. C. Miller, Phys. Rev. 104 (5), 1242 [1956] ; 107 (1), 65 [1957].

2 F. M. Smits, R. C. Miller u. R. L. Batdorf, Halbleiter und Phosphore, Vorträge des Internationalen Kolloquiums Garmisch-Partenkirchen 1956, S. 329. einer Zwischenphase auf der Halbleiteroberfläche wurde durch Untersuchungen verschiedener Autoren ${ }^{1-4}$ weitgehend geklärt.

In Gegenwart von Sauerstoff liegt je nach Quellensubstanz und $\mathrm{O}_{2}$-Menge ein Teil oder aller Phosphor im Reaktionsraum als $\mathrm{P}_{2} \mathrm{O}_{5}$ bzw. $\mathrm{P}_{4} \mathrm{O}_{10}$ vor. Auf der Oberfläche des Siliciums bildet sich eine glasige P-haltige Oxidschicht. Der Einbau des Phosphors ins Silicium läßt sich nicht mehr beschreiben als Austausch zwischen im Festkörper gelösten P. Atomen und solchen im Gasraum, sondern wird durch die Eigenschaften dieser glasigen Zwischenschicht geregelt. Bei gleichen P-Konzentrationen im Gasraum geht der Einbau von Phosphor ins Silicium in Gegenwart von $\mathrm{O}_{2}$ wesentlich schneller vor sich als in reinem $\mathrm{P}_{\mathrm{X}}$-Dampf ${ }^{5,6}$.

Die Gleichgewichtszusammensetzung der Phosphorglas-Schicht, die sich nach einer Vordiffusion in $\mathrm{P}_{2} \mathrm{O}_{5}$-Dampf bei $920{ }^{\circ} \mathrm{C}$, bei einer Nachdiffusion in Inertgas bei verschiedenen Temperaturen einstellt,

3 R. Bullough, R. C. Newmann u. J. Wakefield, Proc. Phys. Soc. London 72 (3), 369 [1958].

4 M. J. Coupland, Proc. Phys. Soc. London 73 (4), 577 [1959].

5 R. P. Donovan u. A. M. Smith, Spring Meeting of the Electrochem. Soc. 13 (1), 108, Oct. 1963.

6 A. B. Kuper u. R. E. Edwards, Spring Meeting of the Electrochem. Soc. 13 (1), 108, May 1964. 
wurde von $\mathrm{Koor}^{7}$ angegeben. Koor bestimmte die Zusammensetzung durch radiochemische Messung des Phosphorgehaltes unter Voraussetzung einer Formel $\left(\mathrm{SiO}_{2}\right)_{n} \cdot \mathrm{P}_{2} \mathrm{O}_{5}$. Dies steht in Übereinstimmung mit Untersuchungen der Reaktion von $\mathrm{P}_{2} \mathrm{O}_{5}$. Dampf mit $\mathrm{SiO}_{2}$-Markierungsschichten ${ }^{8-12}$. FRÄNZ, LANGHeINRICH und Löcherer ${ }^{12}$ konnten zeigen, daß der Einbau von Phosphor aus $\mathrm{P}_{2} \mathrm{O}_{5}$-Dampf in $\mathrm{SiO}_{2}$ im stöchiometrischen Verhältnis $\mathrm{P}_{2} \mathrm{O}_{5}$ erfolgt.

Angaben über die Konzentration von $\mathrm{P}_{2} \mathrm{O}_{5}$ im Diffusionsrohr finden sich bei Koor nicht. Dagegen wurde von SMith und Donovan ${ }^{5}$ ein quantitativer Zusammenhang zwischen dem Schichtwiderstand einer dünnen, bei der Diffusion erhaltenen n-Schicht in p-Silicium und der Konzentration von Phosphor im Trägergas gemessen. Als Phosphorquelle wurde $\mathrm{PH}_{3}$-Gas verwendet und ausreichend $\mathrm{O}_{2}$ für den vollständigen Umsatz zu $\mathrm{P}_{2} \mathrm{O}_{5}$ zugegeben.

Das Ziel der vorliegenden Arbeit war es, nun eine Beziehung herzustellen zwischen der Phosphorkonzentration im Diffusionsraum einerseits und der $\mathrm{Zu}$ sammensetzung des Phosphorglases sowie dem insgesamt vom Silicium aufgenommenen Phosphor andererseits.

Aus radiochemischen Messungen ist bekannt, daß die Gesamtmenge des im Silicium eingebauten Phosphors wesentlich höher sein kann als die durch elektrische Messungen bestimmte ${ }^{7,13}$. Die Hauptmasse des elektrisch inaktiven Phosphors ist dabei in der äußersten Oberflächenschicht von ca. $0,1 \mu \mathrm{m}$ angereichert. Schmidt und Stickler ${ }^{14}$ haben im Elektronenmikroskop (Durchleuchtung) orientierte kompakte Ausscheidungen bis zu $1 \mu \mathrm{m}$ Länge nachgewiesen, die an Hand des Beugungsbildes als SiP identifiziert werden konnten. Zum Verständnis des Mechanismus der Dotierung von Silicium mit Phosphor ist es daher notwendig, auch den elektrisch nicht aktiv eingebauten Phosphor zu erfassen.

\section{Durchführung der Versuche}

Alle Versuche wurden durchgeführt an Riegeln $(3 \mathrm{~mm} \times 10 \mathrm{~mm})$ aus $1 \Omega \mathrm{cm}$ p-Typ Silicium, $150 \mu \mathrm{m}$ stark, einige Sonderversuche mit ganzen Scheiben von

7 E. Koor, J. Electrochem. Soc. 111 (12), 1383 [1964].

8 C. T. Sah, H. Sello u. D. A. Tremere, J. Phys. Chem. Solids 11, 288 [1959].

9 R. B. Allen, H. Bernstein u. A. D. Kurtz, J. Appl. Phys. 31, 334 [1960].

10 M. O. Thurston, J. C. C. Tsai u. K. D. Kang, US Gov. Res. Rep. AD 261 201, March 1961.
$16 \mathrm{~mm}$ Durchmesser. Das Material war einseitig mechanisch poliert, gemessen wurde an der polierten Seite. Vor der Diffusion wurden die Proben durch Kochen in Trichloräthylen, 65-proz. $\mathrm{HNO}_{3}$ und destilliertem deionisiertem Wasser gereinigt und in 2-proz. HF überätzt.

\subsection{Diffusion}

Die für unsere Versuche verwendete Anlage ist in Abb. 1 skizziert. Die Siliciumproben werden von der Ausgangsseite (im Bild links) in den Ofen eingeführt. Die Ofentemperatur war bei allen Versuchen $971{ }^{\circ} \mathrm{C}$, Länge der konstanten Zone $50 \mathrm{~cm}$. Nach dem Einbringen der Proben wird das Rohr zunächst über den Bypasszweig (18 bis 6) mit reinem Trägergas $10 \mathrm{~min}$ durchgespült. Danach wird 5 min lang entweder nur über den Quellenzweig (19 bis 6) allein oder zusammen mit Bypass das Rohr mit Dotierungsstoff gefüllt. Anschließend wird das Rohr beidseitig (Hähne 6 und 5) verschlossen. Das Silicium verbleibt $40 \mathrm{~min}$ in der stehenden dotierten Atmosphäre. Vor der Entnahme wird nochmals 10 min nur über den Bypass gespült.

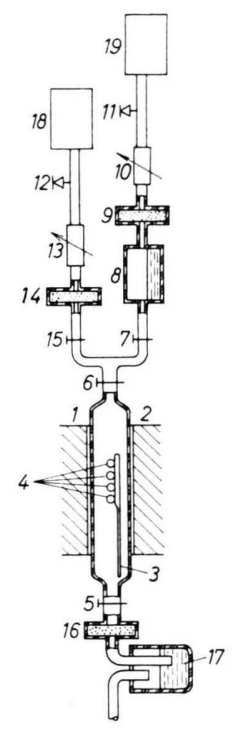

Abb. 1.

Anlage zur Diffusion mit $\mathrm{POCl}_{3}$.

1 Quarzrohr

2 Ofen

3 Schieber

4 Si-Scheiben

$5,6,7,15$ Hähne

8 Quelle

9, 14 Flasche mit Molekularsieb und Staubfilter

16 Flasche mit Molekularsieb

17 Neutralisationsflasche, $20 \% \mathrm{NaOH}$

10, 13 Strömungsmesser

11, 12 Regelventile

18, 19 Gasversorgung

Als Quelle dient ein doppelwandiger, flüssigkeitstemperierter Zylinder von $5 \mathrm{~cm}$ Innendurchmesser. Die Quellentemperatur betrug bei allen Versuchen $21{ }^{\circ} \mathrm{C}$. Das mit $\mathrm{POCl}_{3}$ zu beladende Trägergas strömt auf einer Länge von ca. $30 \mathrm{~cm}$ über eine Flüssigkeitsfläche von ca. $160 \mathrm{~cm}^{2}$. Die Strömungsgeschwindigkeit kann

11 M. O. Thurston, R. B. Menon u. J. C. C. Tsai, US Gov. Res. Rep. 1233-9 Q AD 411 143, May 1963.

12 I. Fränz, W. Langheinrich u. K. H. Löcherer, TelefunkenZtg. 37 (3/4), 194 [1964].

13 P. F. Schmidt u. A. E. Owen, J. Electrochem. Soc. 111, 682 [1964].

14 P. F. Sснмidt u. R. Stickler, J. Electrochem. Soc. 111 (10), 1188 [1964]. 
in beiden Zweigen über die Reduzierventile 12 und 11 unabhängig voneinander eingestellt werden. Als Trägergas dient - wenn nicht ausdrücklich anders angegeben - $\mathrm{O}_{2}$ (einige Sonderversuche $\mathrm{Ar}$ ).

Zur Kontrolle wurden einige Vergleichsdiffusionen im strömenden Gas bei verschiedenen Einstellungen im Quellen- und Bypasszweig gefahren. An einem Teil der Proberiegel wurde eine Nachdiffusion mit verschiedenen Zeiten in einem gesonderten Ofen bei $1020{ }^{\circ} \mathrm{C}$ im $\mathrm{O}_{2}$ Strom durchgeführt. Dabei wurde das während der oben beschriebenen Vordiffusion aufgewachsene P-Glas teils belassen, teils mit 40 -proz. HF abgeätzt.

\subsection{Quellenergiebigkeit}

Zur Messung der Ergiebigkeit der Quelle wurde ein $\mathrm{O}_{2}$-Strom über definierte Zeiten mit verschiedenen Strömungsgeschwindigkeiten durch die Quelle direkt in eine Flasche mit $\mathrm{NaOH}$ geleitet. Wie durch Kontrollmessungen festgestellt wurde, wird $\mathrm{POCl}_{3}$ in dieser Flasche quantitativ umgesetzt. Die Menge des während der Meßzeit durchgelaufenen $\mathrm{POCl}_{3}$ wurde an Hand des in der Lauge gebundenen Chlors bestimmt.

\subsection{Messungen an diffundierten Proben}

Nach der Diffusion wurden nach Abätzen des Phosphorglases der Schichtwiderstand nach der 4-Spitzenmethode $^{15}$ und die Eindringtiefe an Schrägschliffen durch Anätzen und Au-Dekoration des p-n-Überganges bestimmt. An einer Reihe von Proberiegeln mit verschiedenen Schichtwiderständen wurde die RöntgenFluoreszenzintensität in der Cambridge-Mikrosonde unter gleichen Bedingungen gemessen.

Die während der Diffusion aufgewachsene Glasschicht wurde unter dem Mikroskop auf Gleichmäßigkeit, Fehlerfreiheit und Farbe bei fester Apertur untersucht. Die Dicke wurde sowohl an Hand der Farbe als auch an teilweise abgeätzten und mit ca. $100 \AA$ Aluminium bedampften Proben im Interferenzmikroskop bestimmt.

Zur Absolutbestimmung der Glaszusammensetzung wurde an jeweils mehreren einseitig abgeätzten ganzen Scheiben aus 2 verschiedenen Diffusionen $\left(R_{\square}=3,7\right.$ und $6 \Omega / \square$ ) der Gesamtphosphorgehalt kolorimetrisch über einen P-Molybdatkomplex bestimmt. Bei bekanntem Volumen und einer Dichte von $2,4 \mathrm{~g} / \mathrm{cm}^{3}$ für P-haltiges $\mathrm{SiO}_{2}$-Glas ${ }^{12}$ wurde die Zusammensetzung unter Zugrundelegung einer Formel $\left(\mathrm{SiO}_{2}\right)_{n} \cdot \mathrm{P}_{2} \mathrm{O}_{5}$ berechnet. Der Fehler der so gewonnenen Angabe liegt etwa bei $\pm 6 \%$.

Relativmessungen des mittleren P-Gehaltes der Glasschichten über den gesamten Bereich der erzielten Schichtwiderstände wurden über die Intensität der Röntgen-Fluoreszenz in der Mikrosonde durchgeführt. Die Zählrate an abgeätzten Proben gleichen Schichtwiderstandes wurde vom Ergebnis der PhosphorglasMessung abgezogen. Eine Korrektur auf Absorption von Primärelektronen $(20 \mathrm{kV})$ und Fluoreszenzstrahlung im Silicium bzw. im Phosphorglas wurde nicht durchgeführt. Bei bis zu $0,28 \mu \mathrm{m}$ Oxiddicke und einer

15 F. M. Smits, Bell Syst. Techn. J. 37, 711 [1958].
Diffusionseindringtiefe von etwa $1 \mu \mathrm{m}$ sind in den Relativwerten keine wesentlichen Änderungen zu erwarten.

Die Gleichmäßigkeit der Zusammensetzung der Glasschicht wurde an der stärksten $(0,28 \mu \mathrm{m})$ Schicht durch Messung der Ätzgeschwindigkeit bei schrittweiser $\ddot{t}$ zung in einer Lösung von $75 \mathrm{~g} \mathrm{NH}_{4}$ und $35 \mathrm{ml} \mathrm{konz.} \mathrm{HF}$ in $250 \mathrm{ml} \mathrm{H}_{2} \mathrm{O}$ überprüft. Mit dieser Lösung hatten Franz, Langheinrich und Löcherer ${ }^{12}$ die Abhängigkeit der Ätzgeschwindigkeit vom P-Gehalt untersucht.

\section{Ergebnisse}

\subsection{Einstellung der Phosphorkonzentration im Diffusionsrohr}

Die Ergiebigkeit $E(Q)$ der Quelle in P-Atomen/ min in Abhängigkeit von der Strömungsgeschwindigkeit $Q$ in $l / \mathrm{min}$ des über die Quelle geleiteten Trägergases ist in Abb. 2 wiedergegeben. Der Verlauf der Kuve ist leicht zu verstehen. Würde in der Quelle unabhängig von $Q$ stets die thermodynamische Sättigungsdichte $Q_{\mathrm{S}}$ herrschen, so müßte $E(Q)$ linear

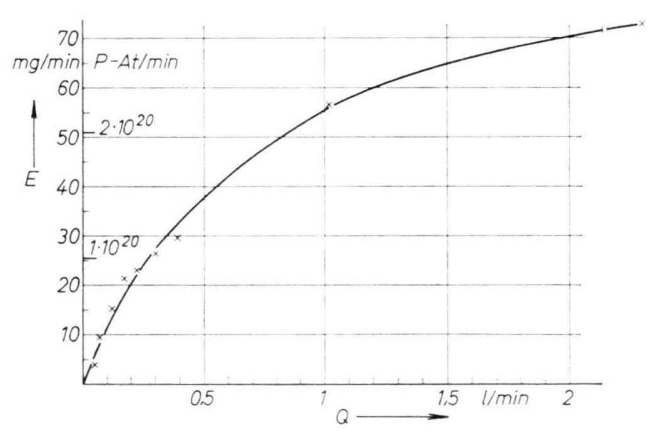

Abb. 2. Ergiebigkeit $E$ der $\mathrm{POCl}_{3}$-Quelle bei $21{ }^{\circ} \mathrm{C}$ in Abhängigkeit vom Quellenstrom $Q$.

mit $Q$ ansteigen. Tatsächlich ist jedoch die Verdampfungsgeschwindigkeit der nachzuliefernden Substanz durch die Oberfläche begrenzt, so daß sich über der Quelle eine Gleichgewichtsdichte $\varrho(Q)<\varrho_{\mathrm{S}}$ einstellt. Die mit dem Trägergas fortgeführte Menge $\mathrm{POCl}_{3}$ ist dann $-\frac{\mathrm{d} M}{\mathrm{~d} t}=Q \cdot \varrho(Q)$ und die durch die Flüssigkeitsoberfläche nachgelieferte Substanzmenge $+\frac{\mathrm{d} M}{\mathrm{~d} t}=a\left(\varrho_{\mathrm{S}}-\varrho(Q)\right)$. Dabei ist $a$ ein kinetischer Faktor, der sowohl von thermodynamischen Daten als auch von der Quellengeometrie, speziell der Oberflächengröße, abhängig ist. Im Gleichgewichtsfall ergibt sich

$$
E(Q)=a \cdot \varrho_{\mathrm{S}} \underset{Q+a}{Q} .
$$


In der experimentell bestimmten Kurve ist $a$ bei kleinem Strömungsvolumen $(Q<0,3 \mathrm{l} / \mathrm{min})$ keine Konstante mehr, sondern fällt $\mathrm{ab}$, was darauf zurückzuführen ist, daß im Quellenraum ein Dichtegradient vorhanden ist, so daß $a$ streng genommen ebenfalls von $Q$ abhängig wird.

Das mit dem Trägergas in die Diffusionszone gelangende $\mathrm{POCl}_{3}$ ist bei den dort herrschenden Temperaturen nicht mehr beständig. Für den thermischen Zerfall sind - ohne Mitwirkung von $\mathrm{O}_{2}$ - die folgenden Reaktionsgleichgewichte bestimmend:

$$
\begin{aligned}
& 5 \mathrm{POCl}_{3} \rightleftarrows 3 \mathrm{PCl}_{5}+\mathrm{P}_{2} \mathrm{O}_{5} \\
& 3 \mathrm{PCl}_{5} \rightleftarrows 3 \mathrm{PCl}_{3}+3 \mathrm{Cl}_{2} .
\end{aligned}
$$

Liegt das Gleichgewicht praktisch auf der rechten Seite, so gilt

$$
5 \mathrm{POCl}_{3} \rightarrow \mathrm{P}_{2} \mathrm{O}_{5}+3 \mathrm{PCl}_{3}+3 \mathrm{Cl}_{2} \text {. }
$$

Die chemische Verbindung, in der die Dotierungs. atome vorliegen, soll zunächst nicht diskutiert werden, da die Verhältnisse in $\mathrm{O}_{2}$ schwieriger zu übersehen sind. In Abschnitt 2.2 wird gezeigt, daß für die Dotierung lediglich der Anteil an $\mathrm{P}_{2} \mathrm{O}_{5}$ wirksam ist.

Beim Übergang in kältere Zonen $\left(<350^{\circ} \mathrm{C}\right.$, Ofenenden) kondensiert $\mathrm{P}_{2} \mathrm{O}_{5}$ in Form eines festen Niederschlages, und zwar nicht nur auf der Ausgangsseite, sondern durch Rückdiffusion auch auf der Eingangsseite. Der Bodenkörper bildet eine zusätzliche Dotierungsquelle und muß, um reproduzierbar arbeiten zu können, unbedingt hinreichend klein gehalten werden.

Die Menge des abgeschiedenen $\mathrm{P}_{2} \mathrm{O}_{5}$ ist

$$
M=\alpha E(Q) t,
$$

wobei $t$ die Durchströmzeit und $\alpha$ ein Faktor ist, der entsprechend dem experimentellen Befund nicht wesentlich kleiner als 1 ist. Andererseits ist die Dichte $Q_{\mathrm{P}}$ der P-Atome - unabhängig davon, in welcher chemischen Verbindung sie vorliegen - im strömenden Gas

$$
\varrho_{\mathrm{P}}=\frac{E(Q)}{Q+B}
$$

mit $Q, B$ als Strömungsgeschwindigkeiten im Quellen- bzw. Bypasszweig.

Zur Begrenzung der Niederschläge bleibt nach (3) und (4) nur die Möglichkeit, die Zeit $t$ klein zu halten, wenn zeitraubende Verfahren, wie häufiger Rohrwechsel oder lange Spülzeiten zwischen den Versuchen vermieden werden sollen.
Schließt man das Rohr ab, nachdem eine gleichmäßige Füllung mit Dotierungsstoff eingestellt ist, so ist zunächst zu erwarten, daß infolge der Temperaturgradienten zwischen der Diffusionszone in der Ofenmitte und den Rohrenden am Ofenausgang der Dotierungsstoff langsam nach außen diffundiert, so $\mathrm{da} ß \varrho_{\mathrm{P}}$ in der Diffusionszone nicht mehr identisch sein muß mit der Dichte nach (4) während des Durchströmens bzw. während des Einfüllvorganges. Die Meßergebnisse haben jedoch gezeigt, daß keine wesentliche Verarmung in der Diffusionszone auftritt. Dieser Befund läßt sich so verstehen, daß die thermische Diffusion aus der heißen Zone kompensiert wird durch eine Rückreaktion an den Rohrenden von $\mathrm{P}_{2} \mathrm{O}_{5}, \mathrm{PCl}_{3}$ und $\mathrm{Cl}_{2}$ (die ja das $\mathrm{Rohr}$ nicht mehr verlassen können) zu $\mathrm{POCl}_{3}$ und damit eine Diffusion von $\mathrm{POCl}_{3}$ zur Rohrmitte.

Die Reaktionsgleichgewichte bei abgeschlossenem Rohr im einzelnen brauchen hier nicht diskutiert zu werden. Experimentell wurden im Bereich hoher $Q_{\mathrm{P}}$ gleiche Ergebnisse im strömenden Gas und bei abgeschlossenem Rohr erzielt. Bei niederem $\varrho_{\mathrm{P}}$ ist die Übereinstimmung experimentell ebenfalls recht gut, wie unter 2.2 an Hand eines Vergleiches mit den Messungen von Sмiтн und Donovan ${ }^{5}$ gezeigt wird. Abweichungen von (4) bei kleinem $\varrho_{\mathrm{P}}$ können demnach zwar nicht völlig ausgeschlossen werden, das Ergebnis der vorliegenden Arbeit kann jedoch mit Sicherheit nur unwesentliche quantitative Verschiebungen erfahren.

Die $\varrho_{\mathrm{P}}$-Werte werden also nach dem unter 1.1 geschilderten Verfahren eingestellt, wobei die Strömungsgeschwindigkeiten $B$ und $Q$ in Gl. (4) den Einstellungen während des Einfüllvorganges entsprechen. Die Größe der eingestellten Phosphordichten liegt dabei in jedem Falle so, daß der vom Silicium aus dem Reaktionsraum herausgezogene Phosphor gegenüber dem im ganzen Rohr vorhandenen Dotierungsstoff zu vernachlässigen ist.

\subsection{Die Abhängigkeit des Schichtwiderstandes von der Dichte der P-Atome im Diffusionsrohr}

Zwischen dem Schichtwiderstand $R_{\square}$ in $\Omega$ pro Flächeneinheit einer dünnen n- (bzw. p) dotierten Schicht auf p- (bzw. n)-dotiertem Substrat und den Ladungsträgerdichten in der Schicht besteht die Beziehung:

$$
\frac{1}{R_{\square}}=\int_{x=0}^{d} \frac{\mathrm{d} \varkappa}{r(x)}=\int_{x=0}^{d}\left[N_{-} \mu_{-}(x)-N_{+} \mu_{+}(x)\right] \mathrm{d} x
$$


$x \quad$ Abstand von der Oberfläche,

$r(x) \quad$ spez. Widerstand in der Tiefe $x$,

$\bar{r} \quad$ mittlerer spez. Widerstand,

$N_{-}, N_{+}$Ladungsträgerdichten,

$\mu_{-}, \mu_{+}$Ladungsträgerbeweglichkeiten,

$d \quad$ Tiefe des p-n-Überganges.

Eine quantitative Auswertung von (5) setzt die Kenntnis des Diffusionsprofiles voraus, ferner müßte der Einfluß von durch die Diffusion erzeugten Gitterstörungen in der äußersten Oberflächenschicht auf die Beweglichkeit berücksichtigt werden. Um die Gesetzmäßigkeiten beim Einbau von Phosphor in Silicium qualitativ zu studieren, genügt es, (5) in der vereinfachten Form

$$
\frac{1}{R_{\square}}=\frac{d}{\bar{r}}=c N_{-} d
$$

$\mathrm{zu}$ schreiben. In den folgenden Überlegungen wird vorausgesetzt, daß die Proportionalitätskonstante $c$ sich nur unwesentlich für verschiedene Diffusionen verändert und $1 / R_{\text {口 }}$ als ein $\mathrm{Ma} \beta$ für die insgesamt durch die Flächeneinheit eindiffundierten P-Atome, die als elektrisch aktive Zentren vorliegen, angesehen werden kann.

Trägt man $R_{\square}$ in Abhängigkeit von den experimentellen Größen $B$ und $Q$ bzw. $B / Q$ auf, so ergibt sich Abb. $3 . R_{\text {口 }}$ steigt mit dem Bypassverhältnis $B / Q$ an, und zwar um so stärker, je größer die Strömungsgeschwindigkeit $Q$ über der Quelle ist.

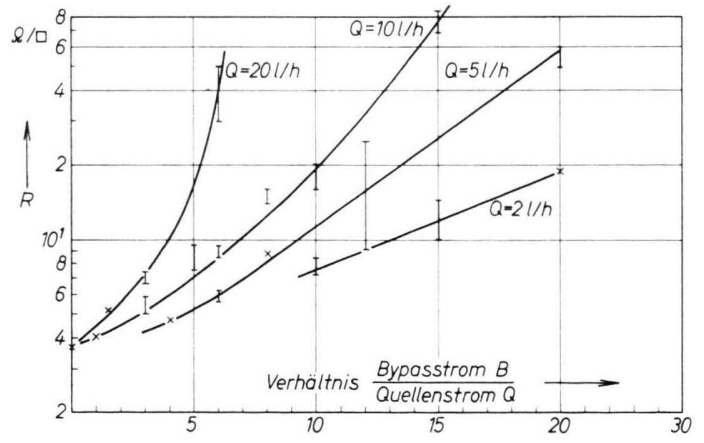

Abb. 3. Riegelwiderstand $R_{\square}$ bei der Vordiffusion $971^{\circ} \mathrm{C}$, in Abhängigkeit vom Bypassverhältnis. Parameter $Q$, Quellenströmung $l / h$.

Die eingetragenen Meßpunkte entsprechen jeweils $1-4$ Versuchen mit 2 bis 3 Riegeln bei $R_{\square}>10 \Omega / \square$ und $5-25$ Versuchen bei $R_{\square} \leqq 10$ $\Omega / \square$. Die Streuung der Werte von Versuch zu Versuch steigt mit wachsendem $B$ und wachsendem $Q$ an, was darauf zurückzuführen ist, daß die Zusammensetzung der beiden Zweige in unserer Anlage bei diesen Versuchen noch nicht ideal war. Ferner ist der Einfluß der Ausgangsoxidhaut von größter Bedeutung. Darauf wird später in der Diskussion unter 3. eingegangen. Die beste Reproduzierbarkeit wurde an Diffusionen ohne Bypass $(B=0, Q=0,33$ l/min) erzielt. Bei 50 Riegeln aus 25 Versuchen lagen alle Einzelmessungen zwischen 3,6 und 3,8 $\Omega / \square$. An 3 Fahrten mit 10-20 ganzen Scheiben wurden die gleichen Werte erzielt (unter Berücksichtigung der anderen Geometrie) wie an einzeln diffundierten Riegeln bei völliger Gleichmäßigkeit der Dotierung über den Scheibenquerschnitt sowie von Scheibe zu Scheibe. Eine Verarmung des Dotierungsstoffes während des Versuches tritt also nicht ein. Ferner ist, wie Kontrollmessungen zeigten, die Eigendotierung des Rohres in allen Fällen zu vernachlässigen.

Trägt man die gemessenen Werte $R_{\square}$ in Abhängigkeit von der Größe $\varrho_{\mathrm{P}}$ nach Gl. (4) auf, so ordnen sich alle Meßpunkte auf einer einzigen Kurve ein, wie in Abb. 4 dargestellt. Die ausgezogene Kurve wurde mit $\mathrm{O}_{2}$ als Trägergas erhalten (s. auch Abb. 3), die gestrichelte mit Ar in beiden Zweigen.

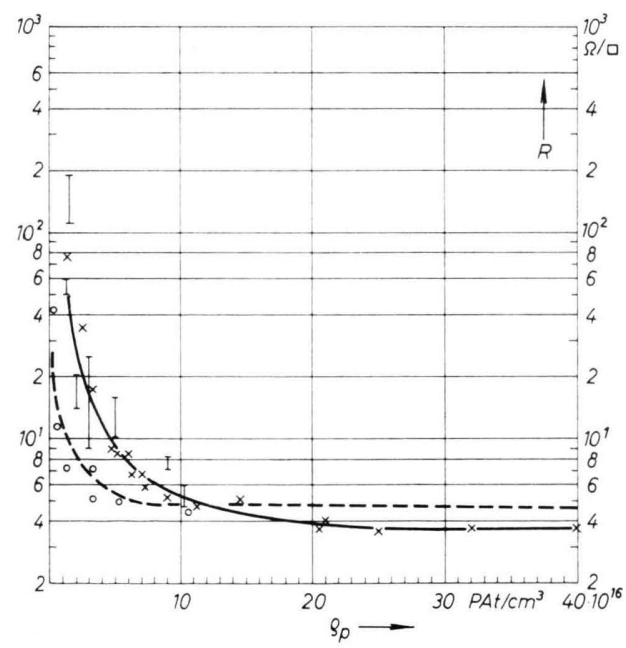

Abb. 4. Riegelwiderstand $R_{\square}$ nach der Vordiffusion mit POCl $_{3}$-Quelle $\left(21^{\circ} \mathrm{C}\right)$ in Abhängigkeit von der Phosphordichte $\varrho_{\mathrm{P}}$ im Diffusionsrohr. $\times \longrightarrow$ Trägergas $\mathrm{O}_{2}$; $0---\rightarrow O$ Trägergas Ar.

Ein Meßpunkt $\circ$ bei $4,8 \Omega / \square$ für $\varrho_{\mathrm{P}}=41 \cdot 10^{16}$ fehlt im Bild.

In Abb. 5 sind die Ergebnisse von Sмітн und Donovan in strömendem $\mathrm{PH}_{3}$ in $\mathrm{N}_{2}\left(1,5 \% \mathrm{O}_{2}\right)$ wiedergegeben. Die Angaben der Autoren in Prozenten wurden unter Zugrundelegung idealer Gasgesetze auf P-Atome pro $\mathrm{cm}^{3}$ umgerechnet. Werden bei den Messungen in Ar aus Abb. 4 die $\varrho_{\mathrm{P}}$-Werte mit $2: 5$ umgerechnet, erhält man die mit $\bigcirc$ eingezeichneten 
Punkte, die sich gut einordnen. Nimmt man also an, $\mathrm{da} ß$ bei einer Diffusion mit $\mathrm{POCl}_{3}$-Dampf der Zerfall in der heißen Zone quantitativ nach (2a) abläuft und lediglich die $\mathrm{P}_{2} \mathrm{O}_{5}$-Komponente für die Diffusion maßgeblich ist, so lassen sich mit zwei völlig verschiedenen Verfahren gleiche Ergebnisse erzielen, sofern die Konzentration von $\mathrm{P}_{2} \mathrm{O}_{5}$ im Diffusionsrohr die gleiche ist. Gleichzeitig kann man dieses Ergebnis als Bestätigung dafür ansehen, daß unsere $\varrho_{\mathrm{P}}$-Werte, wie nach (4) angegeben, im wesentlichen richtig sind.

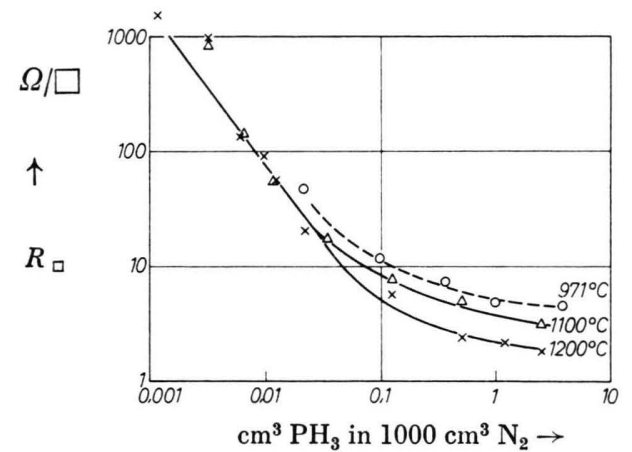

Abb. 5. Schichtwiderstand $R_{\square}$ in Abhängigkeit vom Anteil $\mathrm{PH}_{3}$ in $1000 \mathrm{~cm}^{3} \mathrm{~N}_{2}$ nach 30 min Diffusionszeit, aus Donovan und Sмттн ${ }^{5}$; O diese Arbeit (Abb. 4, Ar). $1 \mathrm{~cm}^{3} \mathrm{PH}_{3} \equiv \varrho_{\mathrm{P}}^{\prime} \approx 2,5 \cdot 10^{16}$.

In der gewählten Darstellung (Abb. 4) läßt sich erkennen, daß offenbar 2 Bereiche mit unterschiedlichem Dotierungsmechanismus für hohe und niedere $R_{\text {口 }}$ existieren. Ein Verständnis der Zusammenhänge kann nur an Hand der in 2.4 beschriebenen Eigenschaften des Phosphorglases gewonnen werden. Ferner muß noch der in elektrisch inaktiver Form vorliegende Phosphor berücksichtigt werden.

\subsection{Inaktiv eingebauter Phosphor und Schichtwiderstand}

In Abb. $6 \mathrm{a}$ ist das Ergebnis von 2 Meßreihen dargestellt, bei denen jeweils an einer Reihe von verschieden diffundierten Proberiegeln (Phosphorglas vorher abgeätzt) die Intensität der RöntGENFluoreszenz des Phosphors in der Mikrosonde gemessen wurde. Die gemessene Impulsrate ist proportional der Menge des insgesamt eingebauten Phosphors. Eine quantitative Auswertung ist nicht ohne weiteres möglich, die grundlegenden Gesetzmäßigkeiten lassen sich bereits an Hand der Relativwerte ablesen.
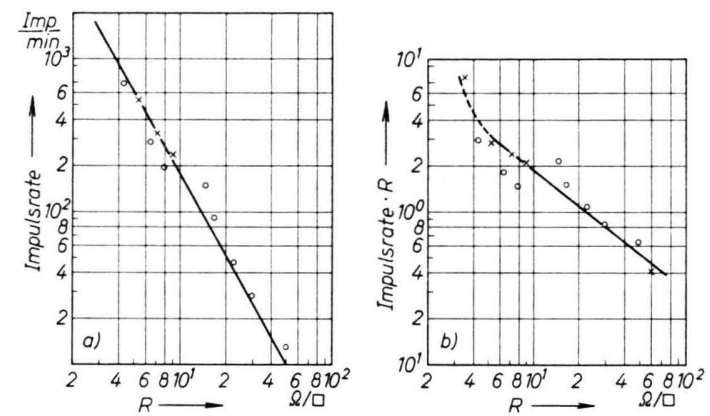

Abb. 6 a. Röntgen-Fluoreszenzintensität des Phosphors in Abhängigkeit vom Schichtwiderstand.

Abb. 6 b. Verhältnis vom Gesamtphosphor zu aktivem Phosphor, Impulsrate $\times R_{\square}$, in Abhängigkeit vom Schichtwiderstand $R_{\square}$.

Aus der Steigung der Kurve in Abb. 6 a folgt

$$
P_{\mathrm{a}}+P_{\mathrm{i}}=\frac{a}{R_{\square}{ }^{1,8}}+b=c P_{\mathrm{a}}^{1,8}+d,
$$

$P_{\mathrm{a}} \quad$ Menge der unter der Flächeneinheit liegen. den P-Atome in elektrisch aktiver Form,

$P_{\mathrm{i}} \quad$ Menge der unter der Flächeneinheit liegen$a, b, c, d$ Konstanten. den P-Atome in elektrisch inaktiver Form,

Innerhalb des untersuchten Bereiches ist kein Anzeichen dafür vorhanden, daß die Kurve zu einer linearen Abhängigkeit von $1 / R_{\square}$ umbiegt, wie sie vorliegen müßte, wenn der gesamte Phosphor in elektrisch aktiver Form vorläge.

Demnach ist auch bei geringer Dotierung (hoher Schichtwiderstand) ein beträchtlicher Anteil von elek trisch inaktivem Phosphor vorhanden, der mit fallendem $R_{\text {口 }}$ stärker ansteigt als die Zahl der elektrisch aktiven Zentren. Das Verhältnis von Gesamtphosphor zu aktivem Phosphor - proportional Impulsrate $\times R_{\square}-$ ist in Abb. $6 \mathrm{~b}$ aufgetragen. Setzt man z. B. bei $100 \Omega / \square$

$$
\frac{P_{\mathrm{i}}+P_{\mathrm{a}}}{P_{\mathrm{a}}}=x,
$$

so ist bei $4 \Omega / \square$ bereits

$$
\frac{P_{\mathrm{i}}+P_{\mathrm{a}}}{P_{\mathrm{a}}}=13 x .
$$

Aus inaktiven Ausscheidungen kann bei einer Nachdiffusion elektrisch aktiver Phosphor abgegeben werden. In Abb. 7 ist das Ergebnis einiger Nachdiffusionen bei $1020{ }^{\circ} \mathrm{C}$ in $\mathrm{O}_{2}$ aufgetragen. Der Schichtwiderstand $R_{\square}$ fällt mit steigender Nachdiffusionszeit. Die Abnahme von $R_{\square}$ mit der Zeit ist um so stärker, je kleiner der Ausgangsschichtwiderstand 
ist. Dieses Ergebnis ist unabhängig davon, ob das während der Vordiffusion aufgewachsene Phosphorglas vor der Nachdiffusion abgelöst wird oder nicht. Zusätzlich elektrisch aktive Störatome, die einen Abfall von $R_{\square}$ bewirken, können demnach nur aus inaktiv eingebautem Phosphor stammen, wie auch aus

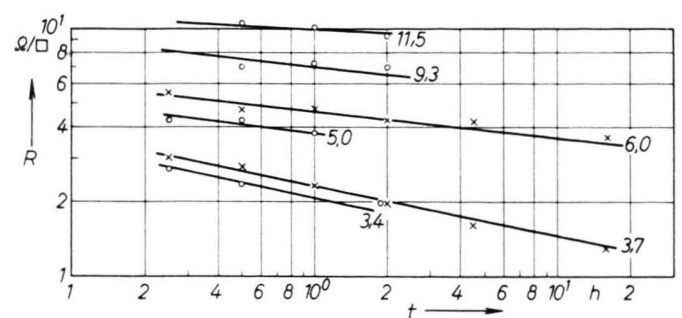

Abb. 7. Schichtwiderstand $R_{\square}$ bei der Nachdiffusion $\left[1020^{\circ} \mathrm{C}\right.$, $\left.\mathrm{O}_{2}+\mathrm{H}_{2} \mathrm{O}\left(83^{\circ} \mathrm{C}\right)\right]$ in Abhängigkeit von der Nachdiffusionszeit $t$. Parameter: Schichtwiderstand nach der Vordiffusion in ० $1 \Omega \mathrm{cm}$ p-Silicium, $\times 0,2 \Omega \mathrm{cm}$ n-Silicium mit Bordiffusion $\left(2,5 \mu \mathrm{m}, c_{0} 5 \cdot 10^{18} / \mathrm{cm}^{3}\right)$.

Tab. 1 ersichtlich ist, in der das Verhalten der Oberflächenkonzentration nach IRviN ${ }^{16}$ während der Nachdiffusion angegeben ist. Die Oberflächenkonzentrationen bleiben bei starker Dotierung zunächst konstant, obwohl Phosphor nach innen abwandert, ohne daß von außen eine Nachlieferung erfolgt. In Übereinstimmung mit dem in Abb. 6 dargestellten Befund ist das inaktive Reservoir um so größer, je geringer der Schichtwiderstand ist.

\subsection{Eigenschaften des während der Diffusion in} $\mathrm{P}_{2} \mathrm{O}_{5}$-Atmosphäre aufwachsenden Phosphorglases

Während der Diffusion wächst auf dem blanken Silicium eine farbige phosphorhaltige Oxidschicht auf. Für das Wachstum dieser Schicht ist der P-Gehalt im Gasraum von ausschlaggebender Bedeutung, wie aus Abb. 8 ersichtlich ist (Trägergas $\mathrm{O}_{2}$ ). Zum Vergleich ist die Kurve $R_{\square}\left(\varrho_{\mathrm{P}}\right)$ (Abb. 4, Trägergas $\mathrm{O}_{2}$ ) mit eingezeichnet.

Die $800 \AA$ bei kleinen $\varrho_{\mathrm{P}}$ entsprechen der Dicke des reinen Oxides, das in $65 \mathrm{~min}$ bei $970{ }^{\circ} \mathrm{C}$ in trockenem $\mathrm{O}_{2}$ auf Silicium aufwächst. Bei hohen Phosphorkonzentrationen werden rund dreimal so dicke Schichten erhalten.

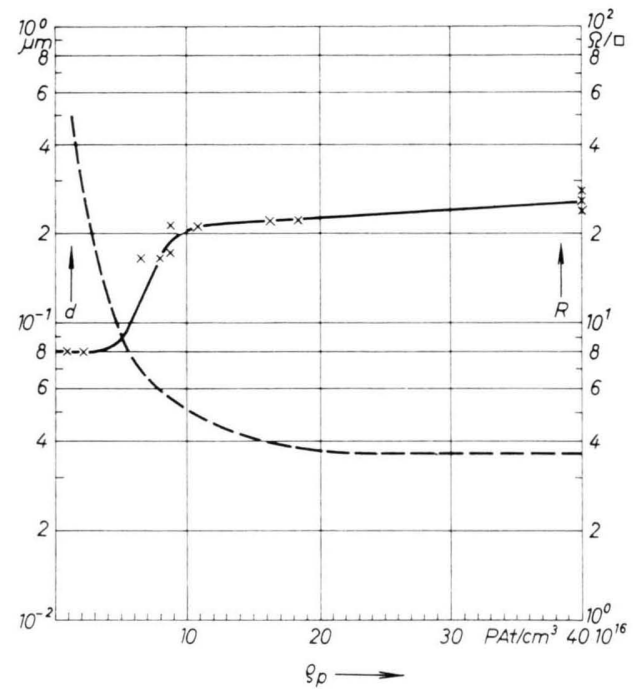

Abb. 8. Dicke der Phosphorglasschicht in Abhängigkeit von $\varrho_{\mathrm{P}} \cdot----$ zum Vergleich $R_{\square}\left(\varrho_{\mathrm{P}}\right)$.

Die bei Diffusion in Ar aufwachsenden Schichten sind für $R_{\square}=5 \Omega / \square$ etwa $800 \AA$ dick, bei $40 \Omega / \square$ nur noch um $50 \AA$. Bei $R_{\square}<6 \Omega / \square$ werden rauhe Schichten mit Blasen und groben Rissen erhalten. Die mit $\mathrm{O}_{2}$ als Trägergas und für $R_{\square}>6 \Omega / \square$ in Ar erhaltenen Oberflächenschichten dagegen sind in allen Fällen glatt, von gleichmäßiger Farbe und fleckenfrei.

\begin{tabular}{|c|c|c|c|c|c|c|}
\hline \multirow{3}{*}{$\begin{array}{c}\text { Nachdiffu- } \\
\text { sionszeit } \\
h\end{array}$} & \multicolumn{6}{|c|}{$\begin{array}{c}\text { Nachdiffusion } 1020^{\circ} \mathrm{C} \text { in } \mathrm{O}_{2}+\mathrm{H}_{2} \mathrm{O}\left(83^{\circ} \mathrm{C}\right) \\
\text { Vordiffusion: nach } 2.1 \mathrm{mit}\end{array}$} \\
\hline & \multicolumn{2}{|c|}{$\mathrm{Ra}=3,7 \mathrm{Ohm} / \mathrm{sq}$} & \multicolumn{2}{|c|}{$\mathrm{Ra}=6,0 \mathrm{Ohm} / \mathrm{sq}$} & \multicolumn{2}{|c|}{$\mathrm{Ra}=9,3 \mathrm{Ohm} / \mathrm{sq}$} \\
\hline & $\mathrm{a}$ & $\mathrm{b}$ & $\mathrm{a}$ & $\mathrm{b}$ & $\mathrm{a}$ & b \\
\hline 0,25 & $3 \cdot 10^{21}$ & $3 \cdot 10^{21}$ & $1,6 \cdot 10^{21}$ & $1,6 \cdot 10^{21}$ & & \\
\hline 0,5 & $2,5 \cdot 10^{21}$ & $2,5 \cdot 10^{21}$ & $1,2 \cdot 10^{21}$ & $1,2 \cdot 10^{21}$ & $4 \cdot 10^{20}$ & $6 \cdot 10^{20}$ \\
\hline 0,7 & & & & & & \\
\hline 1,0 & $2,5 \cdot 10^{21}$ & $2,5 \cdot 10^{21}$ & $8 \cdot 10^{20}$ & $1,2 \cdot 10^{21}$ & $2 \cdot 10^{20}$ & $3 \cdot 10^{20}$ \\
\hline 2,0 & $2,5 \cdot 10^{21}$ & $2,5 \cdot 10^{21}$ & $7 \cdot 10^{20}$ & $1,1 \cdot 10^{21}$ & $1,8 \cdot 10^{20}$ & $2,5 \cdot 10^{20}$ \\
\hline 4,5 & $2,5 \cdot 10^{21}$ & $2,5 \cdot 10^{21}$ & $5 \cdot 10^{20}$ & $1 \cdot 10^{21}$ & & \\
\hline 16,0 & $1,5 \cdot 10^{21}$ & $1,5 \cdot 10^{21}$ & $2 \cdot 10^{20}$ & $3 \cdot 10^{20}$ & & \\
\hline
\end{tabular}

Tab. 1. Oberflächenkonzentration $C_{0}, \mathrm{~cm}^{-3}$, nach der Nachdiffusion unter Annahme einer Verteilung nach a) GAuss-Funktion; b) komplementäre Fehlerfunktion (Auswertung nach Irvis).

16 J. C. Irvis, Bell Syst. Techn. J. 41, 387 [1962]. 
Die Zusammensetzung einer Glasschicht bei $R_{\square}=3,7 \Omega / \square$ (Trägergas $\mathrm{O}_{2}$ ) ist nach einer Messung der Ätzgeschwindigkeit im wesentlichen gleichförmig über die Dicke. Dünne Grenzschichten aus reinem $\mathrm{SiO}_{2}$, wie von $\mathrm{Koor}^{7}$ nachgewiesen, lassen sich mit dieser Methode allerdings nicht erfassen.

Bei der kombinierten Analyse naßchemisch - Mikrosonde ergab sich:

a) Trägergas $\mathrm{O}_{2}$. Bis herauf zu etwat $20 \Omega / \square$ bleibt die Zusammensetzung des Phosphorglases praktisch konstant bei $3,9 \mathrm{SiO}_{2} \cdot \mathrm{P}_{2} \mathrm{O}_{5}$, das entspricht $8 \cdot 10^{21} \mathrm{P}$-Atome $/ \mathrm{cm}^{3}$. Bei höheren Schichtwiderständen enthalten die zugehörigen Schichten wesentlich weniger Phosphor (bei $60-80 \Omega / \square$ etwa $10 \mathrm{SiO}_{2}$ $\left.\cdot \mathrm{P}_{2} \mathrm{O}_{5}\right)$.

b) Trägergas Ar. Wegen der Unsicherheit der Dickenmessung - alle Schichten sind an glatten Stellen unter $800 \AA$ - sind nur grobe Angaben über die Zusammensetzung möglich. Auf dem ansteigenden Ast der $R\left(\varrho_{\mathrm{P}}\right)$-Kurve steigt der $\mathrm{SiO}_{2}$ Anteil von $4: 1$ zu höheren Anteilen $\mathrm{SiO}_{2}$ an. $\mathrm{Zu}$ dem niedersten Schichtwiderstand von $4,6 \Omega / \square$ gehört eine Phosphorglasschicht mit sehr hohem $\mathrm{P}_{2} \mathrm{O}_{5}$ Anteil, der möglicherweise sogar höher ist als der von $\mathrm{SiO}_{2}$.

Zur Klärung der Frage nach der Durchlässigkeit des zu Beginn der Diffusion aufwachsenden Phosphorglases für $\mathrm{P}_{2} \mathrm{O}_{5}$ aus dem Außenraum wurden zwei Sonder-Versuchsreihen mit verschiedener $\varrho_{\mathrm{P}}$ Einstellung durchgeführt. Dabei wurden die Proberiegel nach der üblichen Vorspülzeit $(10 \mathrm{~min})$ und der normalen Einfüllzeit $(5 \mathrm{~min})$ a) im reinen undotierten Gasstrom, b) bei abgeschlossenem Rohr im stehenden dotierten Gas über verschiedene Zeiten in der Diffusionszone belassen. Das Ergebnis ist in Abb. 9 dargestellt. Der Schichtwiderstand bei $t=50$

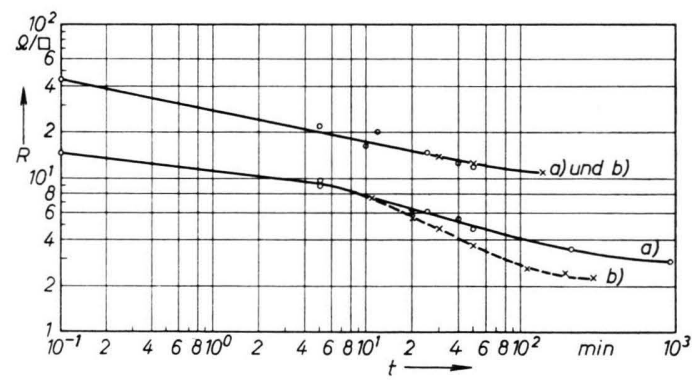

Abb. 9. Schichtwiderstand $R_{\square}$ in Abhängigkeit von der Vordiffusionszeit $t$ nach Beendigung des Einfüllens. a) in strömendem $\mathrm{O}_{2} \mathrm{O}-\mathrm{O}$, b) im stehenden, dotierten Gas (einschließlich 10 min Nachspülzeit $\mathrm{O}_{2}$ ) $\times----\times$. min der Kurven b) entspricht dem Ergebnis mit Standardzeiten nach 1.1. Bei hohem $\varrho_{\mathrm{P}}^{\prime}$ (einige $10^{17}$ P-Atome $/ \mathrm{cm}^{3}$ ) besteht ein deutlicher Unterschied zwischen Kurve a) und b), bei kleinem $\varrho_{\mathrm{P}}\left(\leqq 5 \cdot 10^{16}\right.$ P-Atome $/ \mathrm{cm}^{3}$ ) sind beide Kurven praktisch identisch.

Während der Anfangszeit wächst eine Phosphorglasschicht auf, deren Dicke etwa $1 / 3$ der nach der Standardzeit (1.1) erreichten Dicke (Abb. 8) beträgt. Der aktive Phosphor, der im weiteren Verlauf im Silicium eingebaut wird, kann aus 3 Quellen stammen: a) aus dem anfänglich aufgewachsenen Phosphorglas, b) aus anfänglich im Silicium eingebauten inaktiven Phosphor und c) aus einer Nachlieferung aus dem Gasraum.

Eine Nachlieferung aus dem Gasraum findet nur bei hohen Phosphorkonzentrationen des Gases statt. Das bei geringem $\varrho_{\mathrm{P}}$ bereits zu Beginn aufwachsende Phosphorglas ist, wenn die Proben länger in der mit $\varrho_{\mathrm{P}}$ dotierten Atmosphäre verbleiben, für weiteren Nachschub undurchlässig - jedenfalls mit $\mathrm{O}_{2}$ als Trägergas.

\section{Diskussion}

Bei einer Temperatur um $1000{ }^{\circ} \mathrm{C}$ können zwischen $\mathrm{Si}$ und $\mathrm{P}_{2} \mathrm{O}_{5}$ die folgenden Reaktionen ablaufen:

$$
\begin{aligned}
& 9 \mathrm{Si}+2 \mathrm{P}_{2} \mathrm{O}_{5} \rightarrow 5 \mathrm{SiO}_{2}+4 \mathrm{SiP}, \\
& 5 \mathrm{Si}+2 \mathrm{P}_{2} \mathrm{O}_{5} \rightarrow 5 \mathrm{SiO}_{2}+4 \mathrm{P} .
\end{aligned}
$$

In beiden Fällen entsteht $\mathrm{SiO}_{2}$. Das so entstandene $\mathrm{SiO}_{2}$ oder eine von vornherein vorhandene Oxidhaut reagieren mit gasförmigem $\mathrm{P}_{2} \mathrm{O}_{5}$ unter Bildung eines binären Oxids $\left(\mathrm{SiO}_{2}\right)_{\mathrm{n}} \cdot \mathrm{P}_{2} \mathrm{O}_{5}{ }^{12}$.

Je nach den experimentellen Bedingungen kann das P-haltige Oxid mit der Umgebung reagieren: 1. durch Abgabe von Phosphor an das feste Silicium nach (7) und/oder (8) und 2. durch Aufnahme von $\mathrm{P}_{2} \mathrm{O}_{5}$ aus dem Gasraum.

\subsection{Abgabe von P aus Si}

Schmidt und Stickler ${ }^{14}$ haben bei elektronenmikroskopischen Messungen Ausscheidungen von SiP nachgewiesen, konnten auf Grund ihrer Messungen jedoch nicht entscheiden, ob aller elektrisch inaktiver Phosphor als SiP-Phase vorliegt. Im folgenden wird vorausgesetzt, daß inaktiver Phosphor als Fremdphase vorliegt, da er offenbar im Gitter unbeweglich ist. Inaktiver Phosphor wurde nur in der äußersten Oberflächenschicht gefunden ${ }^{7}{ }^{13}$. 
Aus unseren Untersuchungen ergab sich (Abb. 6), daß auch bei relativ geringer Dotierung inaktiver Phosphor vorhanden ist. Danach ist anzunehmen, daß die Bildung von SiP eine normale Begleiterscheinung der Reaktionen an der Grenzfläche Silicium - Phosphorglas ist und nicht erst bei Überschreiten einer Löslichkeitsgrenze auftritt. Entweder laufen also die Reaktionen (7) und (8) nebeneinander $\mathrm{ab}$, oder es wird primär nur $\mathrm{SiP}$ gebildet, und der elektrisch aktive Phosphor entsteht durch Abgabe von $\mathrm{P}$ aus SiP-Ausscheidungen.

Daß inaktive Fremdphasen sich teilweise oder ganz wieder auflösen können, zeigt das Ergebnis an nachdiffundierten Riegeln (Abb. 7, Tab. 1) und wurde auch von O'Keeffe, Sсhмidt und Stickler ${ }^{17}$ im Elektronenmikroskop beobachtet. Nimmt man plausiblerweise an, daß bei konstanter Temperatur die Auflösungsgeschwindigkeit primär gebildeter Fremdphasen mit wachsender aktiver Oberflächenkonzentration fällt und bei einem Grenzwert von ca. $10^{21} \mathrm{~cm}^{-3}$ (Tab. 1) praktisch gleich Null wird, so wird der starke Anstieg des inaktiven Anteils mit fallendem Schichtwiderstand wie auch das Fallen des Schichtwiderstandes ohne Nachlieferung von Dotierungsstoff von außen verständlich. Die Verhältnisse in der Grenzschicht werden bestimmt durch das Verhältnis von Phosphorangebot, für das die Eigenschaften der Zwischenschicht verantwortlich sind, zur Abwanderung durch Diffusion. Bei gleichem Angebot muß der Anteil an inaktiven Ausscheidungen mit wachsender Temperatur - also steigender Diffusionsgeschwindigkeit - fallen, wie auch von Kоог ${ }^{7}$ beobachtet wurde.

Der Grenzzwert der aktiven Oberflächenkonzentration, über den hinaus kein elektrisch aktiver Phosphor abgegeben wird, ist von der Temperatur abhängig ${ }^{7}$ und stimmt mit durch elektrische Messungen bestimmten Werten der Löslichkeitsgrenze ${ }^{18}$ überein.

\subsection{Die Regelung des Angebotes durch die P-Glasschicht}

Für die Reaktion eines Festkörpers bzw. einer Schmelze mit Gasen sind die Partialdrucke der gasförmigen Reaktionspartner in der Reaktionszone bestimmend.

17 T. W. O'Keefe, P. F. Schmidt u. R. Stickler, J. Electrochem. Soc. $112(8), 878$ [1965].

18 F. A. Trumbore, Bell Syst. Techn. J. 39, 205 [1960].
Um Aussagen zu erhalten, die unabhängig von der speziellen experimentellen Anordnung sind, wurden die vorliegenden Untersuchungen auf die Dichte des Dotierungsstoffes bezogen. Phosphor reagiert mit dem Phosphorglas an der Oberfläche des Siliciums nur, wenn es in Form von $\mathrm{P}_{2} \mathrm{O}_{5}$ vorliegt. Da die Zerfallsreaktionen des $\mathrm{POCl}_{3}$ in $\mathrm{O}_{2}$ schwer übersehbar sind, wurden der Genauigkeit halber die Dichten aller $\mathrm{P}$-Atome angegeben; in Inertgas ist dann $\varrho_{\mathrm{P}}^{\prime}\left(\mathrm{P}_{2} \mathrm{O}_{5}\right)=\frac{2}{5} \varrho_{\mathrm{P}}$, in $\mathrm{O}_{2}$ ist $\varrho_{\mathrm{P}}^{\prime}\left(\mathrm{P}_{2} \mathrm{O}_{5}\right)$ wahrscheinlich etwas höher, wenn auch nicht wesentlich, wie ein Vergleich der Kurven in Abb. 4 zeigt.

Abb. 10 zeigt nach $\mathrm{KooI}^{7}$ die Gleichgewichtszusammensetzung des P-Glases in Abhängigkeit von der Temperatur - bei hoher $\mathrm{P}_{2} \mathrm{O}_{5}$-Dichte - in das Phasendiagramm $\mathrm{SiO}_{2}-\mathrm{P}_{2} \mathrm{O}_{5}$ eingezeichnet. Zusätzlich wird mit $\bigcirc$ eine Messung von $F_{R} \ddot{A N Z}$ u. a. ${ }^{12}$ und mit $\times$ das Ergebnis dieser Arbeit $\left[\right.$ für $\varrho_{\mathrm{P}}^{\prime}\left(\mathrm{P}_{2} \mathrm{O}_{5}\right)$ $\approx 10^{17} \mathrm{P}$-Atome $\left./ \mathrm{cm}^{3}\right]$ mit angegeben. Alle Messungen liegen teils auf, teils nahe der Liquiduskurve. Bei unserer Messung ist die Gleichgewichtszusammensetzung nur angenähert erreicht.

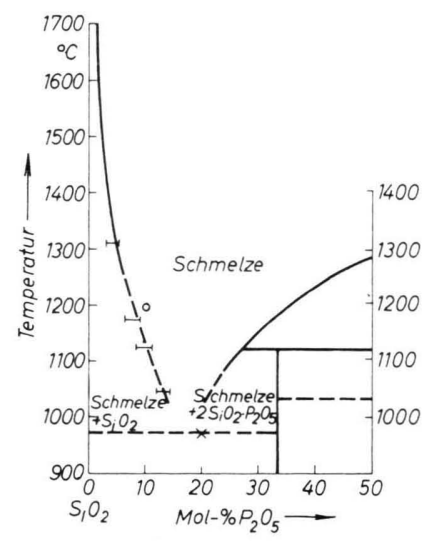

Abb. 10. Phasendiagramm des Systems $\mathrm{SiO}_{2}-\mathrm{P}_{2} \mathrm{O}_{5}{ }^{19}$.

Wie die Temperaturabhängigkeit ist auch die Abhängigkeit der Glaszusammensetzung und der Trägerkonzentration im $\mathrm{Si}$ an Hand des Zustandsdiagramms leicht zu verstehen. Dabei kann vorausgesetzt werden, daß die Diffusionsgeschwindigkeit von Phosphor in einer Schmelze groß ist gegenüber der Diffusionsgeschwindigkeit durch festes $\mathrm{SiO}_{2}$.

Sei $C_{\mathrm{s}}$ die $\mathrm{P}_{2} \mathrm{O}_{5}$-Konzentration im Glas, die mit $Q_{\mathrm{P}}{ }^{\prime}$ und $\mathrm{O}_{2}$ im Gasraum im Gleichgewicht steht, $C_{\mathrm{L}}$

19 T. Y. Tien u. F. A. Hummel, J. Amer. Ceram. Soc. 45, 422 [1962]. 
die der Diffusionstemperatur entsprechende Konzentration auf der Liquiduskurve. Für $\varrho_{\mathrm{P}}{ }^{\prime}$-Werte, die höher liegen, als dem Knick in der $R_{\square}\left(\varrho_{\mathrm{P}}\right)$-Kurve (Abb. 4) entspricht, entsteht während des Einfüllens ein Glas mit der $\mathrm{P}_{2} \mathrm{O}_{5}$-Konzentration $C_{\mathrm{A}}$; $C_{\mathrm{L}}<C_{\mathrm{A}}<C_{\mathrm{S}}$.

An der Grenze zum Silicium reagiert $\mathrm{P}_{2} \mathrm{O}_{5}$ mit dem Silicium unter Bildung von $\mathrm{SiO}_{2}$, das von der Glasschmelze gelöst wird. Dabei sinkt die Konzentration von $\mathrm{P}_{2} \mathrm{O}_{5}$ im Glas ab. Bei Erreichen von $C_{\mathrm{L}}$ ist festes $\mathrm{SiO}_{2}$ neben der Schmelze beständig, es bildet sich eine zunächst nur sehr dünne Schicht aus festem $\mathrm{SiO}_{2}$ an der Grenze Silicium/Glas aus. (Eine solche Grenzschicht wurde von Koor ${ }^{7}$ nachgewiesen.)

Entsprechend dem Wachstum der festen $\mathrm{SiO}_{2}$ Schicht wird der Nachschub von Phosphor aus dem hoch phosphorhaltigen Glas einschneidend gebremst. Gleichzeitig mit einer Verarmung von Phosphor an der Grenze zum Silicium bildet sich an der Grenze zum Gasraum eine stark phosphorhaltige Schicht, die proportional zu $C_{\mathrm{S}}-C_{\Lambda}$ zu wachsen scheint (Abb. 8).

Dadurch steigt die Konzentration in der Schmelze, festes $\mathrm{SiO}_{2}$ kann wieder aufgelöst werden.

Bei ungenügendem Nachschub aus dem Gasraum $\left(C_{\mathrm{A}}<C_{\mathrm{L}}\right)$ kann die feste $\mathrm{SiO}_{2}$-Schicht relativ dick werden, das Angebot von Phosphor ans Silicium wird nicht mehr durch Diffusion durch eine Schmelze, sondern durch Diffusion im festen $\mathrm{SiO}_{2}$ bestimmt. Der steile Anstieg der $R_{\square}\left(\varrho_{\mathrm{P}}\right)$-Kurve bei kleinen $\varrho_{\mathrm{P}}$ erklärt sich dann daraus, daß mit fallendem Angebot gleichzeitig die bremsende $\mathrm{SiO}_{2}$-Schicht ansteigt.

Wird das Angebot durch Diffusion durch festes $\mathrm{SiO}_{2}$ bestimmt, besteht eine starke Abhängigkeit von der Oxidhaut vor Beginn der Behandlung, was die Streuung der Schichtwiderstände bei kleinem $\varrho_{\mathrm{P}}$ verständlich macht.

Wie die geringeren Schichtdicken bei Diffusion in Inertgas zeigen, läuft die Reaktion von $\mathrm{P}_{2} \mathrm{O}_{5}$ mit $\mathrm{Si}$ wesentlich langsamer als mit $\mathrm{SiO}_{2}$. Bei hohen $\mathrm{P}_{2} \mathrm{O}_{5}$-Konzentrationen in Inertgas bilden sich instabile Schichten mit extrem hohem $\mathrm{P}_{2} \mathrm{O}_{5}$-Anteil. Möglicherweise entsteht dabei an der Grenze zum Gasraum festes $2 \mathrm{SiO}_{2} \cdot \mathrm{P}_{2} \mathrm{O}_{5}$, was erklären könnte, da $\Omega$ in Ar bei hohem $\varrho_{\mathrm{P}}$ etwas höhere Schichtwiderstände als in $\mathrm{O}_{2}$ gemessen wurden.

Für die Durchführung der chemischen Analysen habe ich Herrn Dr. A. Pink und Herrn Dr. L. Schoepp, für die Röntgen-Fluoreszenzmessungen Herrn Dipl.-Phys. H. Rehme zu danken. Ferner bin ich Herrn Dr. H. Christ, Herrn Dr. E. Sirtl und Herrn Dr. H. Ullrich für wertvolle Diskussionen und Frau M. Brandhuber für Hilfe bei den experimentellen Arbeiten zu Dank verpflichtet. 